\title{
NSTE-ACS ESC Guidelines Recommend Prasugrel as the Preferred P2Y12 Inhibitor: A Contrarian View
}

\author{
Denis Angoulvant ${ }^{1,2,3} \cdot$ Pierre Sabouret $^{1,2,3}$ (]) $\cdot$ Michael P. Savage ${ }^{1,2,3}$
}

Accepted: 19 February 2021 / Published online: 6 March 2021

(c) The Author(s) 2021

\begin{abstract}
In the 2020 European Society of Cardiology guidelines on non-ST-segment elevation acute coronary syndromes (NSTEACS), the experts proposed to put an end to the equipoise of ticagrelor and prasugrel in addition to aspirin in patients with NSTE-ACS who proceed to percutaneous coronary intervention (PCI). They gave a strong level of recommendation (IIa) in favor of prasugrel over ticagrelor in these patients. We challenge this proposition, which was mainly driven by the results of ISAR-REACT 5, an open-label prospective head-to-head study of a prasugrel-based strategy compared with a ticagrelor-based strategy in patients with ACS undergoing PCI. In addition to the methodological concerns regarding the ISAR-REACT 5 study, we also question this decision in light of the ISAR-REACT 5 diabetes mellitus subgroup analysis and previous studies and meta-analysis that showed no difference between ticagrelor and prasugrel in patients with ACS. Although we agree with the "one size does not fit all" concept for antiplatelet regimens in patients with ACS who proceed to PCI, we believe that the decision to strongly favor prasugrel was premature and not supported enough by the ISAR-REACT 5 results. In our opinion, equipoise remains between the ticagrelor- and prasugrel-based strategies and more data are needed to settle the debate.
\end{abstract}

\section{Key Points}

We disagree with the latest European Society of Cardiology guidelines about the preferred choice concerning P2Y12 inhibitors. Although the ISAR-REACT 5 data support the "one size does not fit all" concept, we believe that the guidelines do not provide sufficient evidence to support a preferred drug or strategy.

Giving a level-IIa recommendation for prasugrel over ticagrelor in patients with NSTE-ACS undergoing PCI seems overrated. In our opinion, equipoise remains, and more data are needed to settle the doubt between these two strategies.

Denis Angoulvant

d.angoulvant@chu-tours.fr

1 Cardiology Department, Loire Valley Cardiovascular Collaboration \& EA4245 T2i, CHRU de Tours \& Tours University, 10, boulevard Tonnellé, Tours 37000, France

2 College National des Cardiologues Français, 13, Rue Niepce, Paris 75014, France

3 Sidney Kimmel Medical College at Thomas Jefferson University, Thomas Jefferson University Hospital, Philadelphia, PA, USA
During the recent European Society of Cardiology (ESC) congress, the new 2020 guidelines on non-ST-segment elevation acute coronary syndromes (NSTE-ACS) were presented and concomitantly published in the European Heart Journal [1]. This comprehensive guideline proposed that "Prasugrel should be preferred over ticagrelor for NSTEACS patients who proceed to PCI [percutaneous coronary intervention]," with a level IIa recommendation grade [1].

This recommendation was based largely on data from ISAR-REACT 5 [2], a single open-label study. This trial was an investigator-initiated study that randomized 4018 patients with ACS in a head-to-head comparison of prasugrel-based and ticagrelor-based P2Y12 inhibition strategies in addition to low-dose aspirin. Although the investigator's hypothesis was a superiority of ticagrelor over prasugrel in patients with ACS undergoing PCI, the results showed a significant reduction of the composite primary endpoint (death, myocardial infarction [MI], and stroke) in the prasugrel strategy group ( 6.9 vs. $9.3 \% ; p=0.006$ ), with a similar risk of major bleeding (4.8 vs. $5.4 \% ; p=0.46$ ) at 12-month follow-up [2].

Interpretation of these data is complex since ISARREACT 5 enrolled two types of patients with ACS 
(ST-segment elevation [STE]-ACS and NSTE-ACS) and compared two treatment strategies: one with a loading dose before PCI (ticagrelor) and one with a loading dose after PCI (prasugrel).

Following these results, the experts rightly questioned the "one size fits all" approach of the ticagrelor strategy. They not only proposed that prasugrel should be favored over ticagrelor but also downgraded the recommendation to administer a P2Y12 inhibitor loading dose before $\mathrm{PCI}$ in patients with NSTE-ACS. In our opinion, the hypothetical superiority of prasugrel over ticagrelor promoted by the ESC guidelines should be challenged.

Indeed, in addition to the numerous and acknowledged methodological limitations of an open-label trial, we also note that the rate of patients not discharged on the allocated P2Y12 inhibitor was higher with ticagrelor than with prasugrel, and that only $60 \%$ of patients who discontinued the investigated P2Y12 inhibitor (15\% with ticagrelor and $12 \%$ with prasugrel) switched to either clopidogrel $(\sim 50 \%)$ or the alternative study drug, leading to more patients in the ticagrelor arm left with aspirin monotherapy in the early post-ACS period.

By 1 year, approximately one-third of the patients were not receiving the assigned study drug. In addition, events were mainly ascertained through phone contact. This raises questions since the outcome results were primarily driven by a difference in self-reported MI, whereas there was no difference in mortality. One may also question the observation that an antiplatelet agent induced fewer ischemic events without increasing bleeding events. In the prespecified platelet inhibition substudy of ISAR-REACT 5, the investigators concluded that a prasugrel-based strategy was associated with enhanced platelet inhibition compared with ticagrelor, which may contribute to its greater reduction in ischemic events [3]. They observed no significant association between adenosine diphosphate-induced platelet aggregation and bleeding but did not provide any pharmacologic insight or hypothesis to discuss this counterintuitive observation.

Controversy may also stem from the recently published prespecified ISAR-REACT 5 substudy of ticagrelor versus prasugrel efficacy according to diabetic status. In this subgroup of patients with high ischemic risk and poor antiplatelet response, the ISAR-REACT 5 investigators observed that the efficacy of prasugrel and ticagrelor in reduction of ischemic events (a composite of death, MI, or stroke) up to 1 year after randomization was comparable. As in the main trial results, there was no difference between antiplatelet strategies regarding bleeding risk in patients with diabetes mellitus (DM). There was no baseline characteristic imbalance between groups, nor was there any biological or pharmacological data to explain these findings. It has been previously suggested that DM may interfere with drug pharmacokinetics, leading to less biotransformation of proactive drugs, such as prasugrel, to their active compound, a phenomenon less likely to affect ticagrelor as it does not require metabolism for activity [4]. Although the discrepancy between results from patients with and without DM in the ISAR-REACT 5 trial remains exploratory, it fuels concern within the scientific community regarding the "prasugrel does it better" hypothesis.

One may notice that the results of ISAR-REACT 5 are not in keeping with those of the previous PRAGUE-18 trial, which found no difference between ticagrelor and prasugrel in patients with STE-ACS for ischemic events-with similar rates of MI (3.0 vs. $2.5 \% ; p=0.611)$, stent thrombosis (1.1 vs. $1.5 \% ; p=0.535)$, and stroke ( 1.1 vs. $0.7 \% ; p=0.423)$ or for major bleeding events ( 0.9 vs. $0.7 \% ; p=0.754$ ) [5]. Both cardiovascular mortality ( 3.3 vs. $3.0 \% ; p=0.769)$ and all-cause mortality ( 4.7 vs. $4.2 \% ; p=0.654)$ were similar at 12-month follow-up. The trial was prematurely stopped because of futility. Notably, a significant amount of crossover was observed for economic reasons. Indeed, about $34 \%$ of patients receiving prasugrel and $44 \%$ of those receiving ticagrelor were financially motivated to switch to clopidogrel, a less expensive drug. The higher discontinuation rate with ticagrelor was attributed to selective discrimination in favor of prasugrel (some recruiting centers provided reimbursements for prasugrel only). Overall, in the PRAGUE-18 trial, investigational drugs elevated costs, and selective reimbursement constraints led to a high incidence of switching to clopidogrel, precluding a reliable comparison of the two drugs. The TOTAL trial, which compared the clinical efficacy of clopidogrel versus ticagrelor and prasugrel, reported opposing findings, as ticagrelor was associated with a $35 \%$ relative risk reduction in the rate of 1 -year major adverse cardiovascular events compared with prasugrel $(p=0.02)$ in an observational subgroup analysis [6]. In addition, ticagrelor demonstrated benefits over clopidogrel in a large spectrum of patients with ACS in the PLATO trial [7]. PLATO was by far the largest trial of the newer P2Y12 inhibitors, with over 18,000 patients. Importantly, at 12 months, mortality was significantly lower with ticagrelor than with clopidogrel ( 4.5 vs. $5.9 \% ; p<0.001)$. In contrast, a similar significant mortality benefit has not been demonstrated with prasugrel.

A recent well-conducted meta-analysis reported similar clinical efficacy and safety for prasugrel and ticagrelor in patients with ACS (Fig. 1) [8]. Of note, in this meta-analysis, prasugrel seemed to be more beneficial at 30 days, but these results were mostly driven by observational studies and the benefit was not significant in the subset of randomized clinical trials. In addition, although ISAR-REACT 5 is the only sizable head-to-head comparison of ticagrelor and prasugrel, it enrolled 4018 patients and resulted in 321 endpoint events. The TRITON and PLATO trials, which enrolled similar numbers of patients, showed comparable treatment effects for prasugrel and ticagrelor compared with 


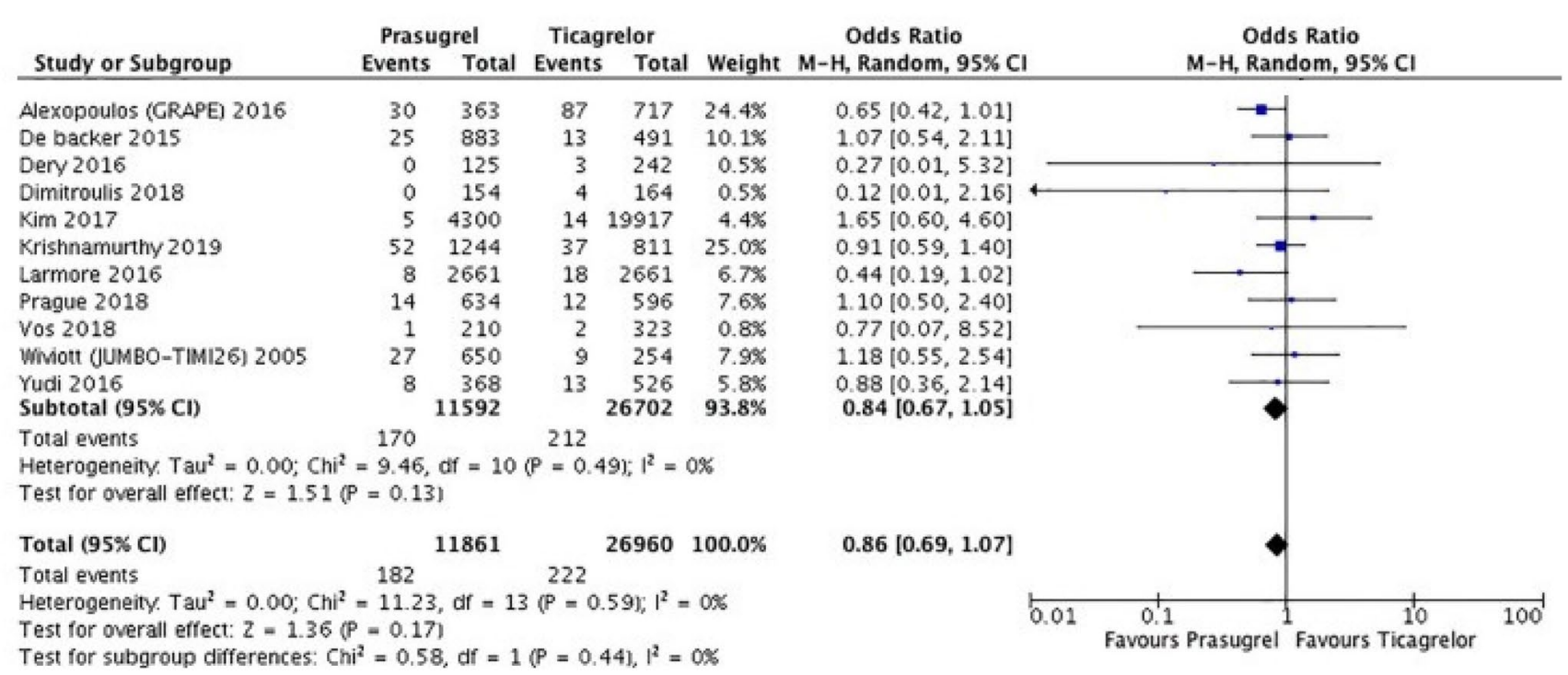

Fig. 1 Forest plot for major adverse cardiovascular events showing individual and pooled relative risks for studies comparing prasugrel and ticagrelor at 1-year follow-up. Reproduced from Ullah et al. [8] with permission. $C I$ confidence interval, $M-H$ Mantel-Haenszel

clopidogrel; these two trials constitute a database of over 32,000 patients and over 3300 endpoint events [7, 9]. In the totality of data, then, the much larger dataset from blinded trials showed no suggestion of the superior treatment effect of prasugrel over ticagrelor that was observed in the smaller ISAR-REACT study.

Cardiologists have several alternatives in terms of drug choices, antithrombotic combinations, and prescription durations. The 2020 ESC guidelines on NSTE-ACS provide efficient tools to individualize both drug combinations and durations, helping physicians address the challenging prediction of ischemic recurrence and bleeding events.

Although the ISAR-REACT 5 data support the "one size does not fit all" concept, we believe that they do not provide sufficient evidence to support a preferred drug or strategy.

Giving a level-IIa recommendation for prasugrel over ticagrelor in patients with NSTE-ACS undergoing PCI seems overrated; in our opinion, equipoise remains. We believe that more data are needed to settle the debate about these two strategies.

\section{Declarations}

Conflict of interest Denis Angoulvant, Pierre Sabouret, and Michael P. Savage have no potential conflicts of interest that might be relevant to the contents of this manuscript.
Author Contributions All three authors contributed to the development of this manuscript.

Funding No external funding was used in the preparation of this manuscript.

Open Access This article is licensed under a Creative Commons Attribution-NonCommercial 4.0 International License, which permits any non-commercial use, sharing, adaptation, distribution and reproduction in any medium or format, as long as you give appropriate credit to the original author(s) and the source, provide a link to the Creative Commons licence, and indicate if changes were made. The images or other third party material in this article are included in the article's Creative Commons licence, unless indicated otherwise in a credit line to the material. If material is not included in the article's Creative Commons licence and your intended use is not permitted by statutory regulation or exceeds the permitted use, you will need to obtain permission directly from the copyright holder. To view a copy of this licence, visit http://creativecommons.org/licenses/by-nc/4.0/.

\section{References}

1. Collet J-P, Thiele H, Barbato E, Barthélémy O, Bauersachs J, Bhatt DL, et al. 2020 ESC Guidelines for the management of acute coronary syndromes in patients presenting without persistent ST-segment elevation. Eur Heart J. 2020. https://doi. org/10.1093/eurheartj/ehaa575.

2. Schüpke S, Neumann F-J, Menichelli M, Mayer K, Bernlochner I, Wöhrle J, et al. Ticagrelor or prasugrel in patients with acute coronary syndromes. N Engl J Med. 2019;381:1524-34. 
3. Mayer K, Bongiovanni D, Karschin V, Sibbing D, Angiolillo DJ, Schunkert H, et al. Ticagrelor or prasugrel for platelet inhibition in acute coronary syndrome patients: The ISAR-REACT 5 Trial. J Am Coll Cardiol. 2020;76:2569-71.

4. Gravel S, Chiasson J-L, Turgeon J, Grangeon A, Michaud V. Modulation of CYP450 activities in patients with type 2 diabetes. Clin Pharmacol Ther. 2019;106:1280-9.

5. Motovska Z, Hlinomaz O, Kala P, Hromadka M, Knot J, Varvarovsky I, et al. 1-year outcomes of patients undergoing primary angioplasty for myocardial infarction treated with prasugrel versus ticagrelor. J Am Coll Cardiol. 2018;71:371-81.

6. Welsh RC, Sidhu RS, Cairns JA, Lavi S, Kedev S, Moreno R, et al. Outcomes among clopidogrel, prasugrel, and ticagrelor in stelevation myocardial infarction patients who underwent primary percutaneous coronary intervention from the TOTAL trial. Can J Cardiol. 2019;35:1377-85.
7. Wallentin L, Becker RC, Budaj A, Cannon CP, Emanuelsson H, Held C, et al. Ticagrelor versus clopidogrel in patients with acute coronary syndromes. N Engl J Med. 2009;361:1045-57.

8. Ullah W, Ali Z, Sadiq U, Rafiq A, Khan S, Mamas MA, et al. Meta-analysis comparing the safety and efficacy of prasugrel and ticagrelor in acute coronary syndrome. Am J Cardiol. 2020;132:22-8.

9. Wiviott SD, Braunwald E, McCabe CH, Montalescot G, Ruzyllo W, Gottlieb S, et al. Prasugrel versus clopidogrel in patients with acute coronary syndromes. N Engl J Med. 2007;357:2001-15. 